\title{
A New Mass Measure and a Simplification of Modern Physics that Make Gravity Predictions Independent of G
}

\author{
Espen Gaarder Haug* \\ Norwegian University of Life Sciences
}

February 1, 2019

\begin{abstract}
Recent experimental research has shown that mass is linked to Compton periodicity. We suggest a new way to look at mass: Namely that mass at its most fundamental level can simply be seen as reduced Compton frequency over the Planck time. In this way, surprisingly, neither the Planck constant nor Newton's gravitational constant are needed to observe the Planck length, nor in any type of calculation or gravitational predictions. The Planck constant is only needed when we want to convert back to the more traditional and we would say arbitrary mass measures such as kg. The theory gives the same predictions as Einstein's special relativity theory, with one very important exception: anything with mass must have a maximum velocity that is a function of the Planck length and the reduced Compton wavelength. For all observed subatomic particles, such as the electron, this velocity is considerably above what is achieved in particle accelerators, but always below the speed of light. This removes a series of infinity challenges in physics. The theory also offers a way to look at a new type of quantum probabilities. As we will show, a long series of equations become simplified in this way.

Further Newton's gravitational constant $G$ is clearly not needed for gravity calculations or predictions; it is the Planck length and the speed of light (gravity) that are essential for gravity, and both can be measured easily with no knowledge of $G$.
\end{abstract}

Key words: Mass, Compton frequency, Planck length, Planck mass, Newton's gravitational constant, Schwarzschild radius.

\section{Introduction}

In section 2, we show that the $\mathrm{kg}$ definition of mass actually should be seen as the reduced Compton frequency ratio of the mass in question. In section 3, we introduce our new mass measure, which is the Compton frequency of the mass in question over one Planck second. In section 4, we show how this is related to relativistic principles. In section 5, we discuss a new type of quantum probabilities and also light. In section 6 , we look at the relativistic energy momentum relation. In section 7 , we show that there exist several Newton-like gravitational formulas with very different gravitational constants that all give the same predictions as Newton (in the case of slow-moving objects). In section 8, we show how to find the Planck length from scratch in a Cavendish apparatus only relying on Newton's insight that the gravitational force follows the inverse square law, but we do not need Newton's gravitational constant. In section 9, we summarize why Newton's gravitational constant just is a composite constant.

\section{Mass as kg and its Connection to Compton Frequency Ratio}

A rest-mass in terms of $\mathrm{kg}$ (or pound) can mathematically be described as

$$
m=\frac{\hbar}{\bar{\lambda}} \frac{1}{c}
$$

The formula is well-known, and is basically the Compton wavelength formula [1] rearranged to get mass on the left side. The formula contains the Planck constant, the reduced Compton wavelength of the particle in question, and the speed of light. Based on this, the mass of an electron can be found simply by measuring the reduced Compton wavelength of the particle as has been done [2], and then inputting that into the formula above. That is to say, the mass of a particle is clearly related to the reduced Compton wavelength of matter. Still, the formula does not seem to give much deeper intuition. The reduced Compton wavelength of one $\mathrm{kg}$ is

*e-mail espenhaug@mac.com. Thanks to Victoria Terces for helping me edit this manuscript. 


$$
\bar{\lambda}=\frac{\hbar}{m c}=\frac{\hbar}{1 \mathrm{~kg} \times c}=\frac{\hbar}{c} \approx 3.52 \times 10^{-43} \mathrm{~m}
$$

This is much shorter than the Planck length. We do not think one $\mathrm{kg}$ has a reduced Compton wavelength itself, as it is a massive composite structure of an enormous amount of atoms that again consist of many fundamental particles. However, the theoretical reduced Compton wavelength of one $\mathrm{kg}$ is directly linked to the reduced Compton wavelength of all the subatomic particles, of which the one kg clump of matter consists. The relation is simply

$$
\bar{\lambda}_{1 k g}=\frac{\bar{\lambda}_{e}}{\frac{1 \mathrm{~kg}}{m_{e}}}=\bar{\lambda}_{e} \times m_{e} \approx 3.52 \times 10^{-43} \mathrm{~m}
$$

where $\bar{\lambda}_{1 \mathrm{~kg}}$ is the theoretical reduced Compton wavelength of a composite mass unit that we use as the mass standard unit (in this case a kg), $\bar{\lambda}_{e}$ is the reduced Compton wavelength of an elementary particle (in this case an electron), and $m_{e}$ is the mass of the electron in terms of $\mathrm{kg}$. The well-known reduced Compton wavelength formula is

$$
\bar{\lambda}=\frac{\hbar}{m c}
$$

This means that if we know the mass in terms of weight ( $\mathrm{kg}$ or pound for example), then we know the reduced Compton wavelength, even if it should be a "hypothetical" Compton wavelength for a composite mass. The reduced Compton frequency of elementary particles and composites of them must be related by

$$
\frac{c}{\bar{\lambda}_{1 k g}}=\frac{1 k g}{m} \frac{c}{\bar{\lambda}}=N \frac{c}{\bar{\lambda}}
$$

where $N$ is the number of this elementary particle in the composite mass. So, the reduced Compton frequency in a composite mass is simply the sum of reduced Compton frequencies in the elementary particles from which the composite mass is built; Compton frequencies are simply additive. This is all elementary, but rather important to understand as we take the next steps in the analysis.

From the reduced Compton length formula 4, we can easily find the reduced Compton frequency of one kg, which must be

$$
\frac{c}{\frac{\hbar}{1 \mathrm{~kg} \times c}}=\frac{c^{2}}{\hbar} \approx 8.52 \times 10^{50} \text { times per second }
$$

This also means the Planck constant is equal to

$$
\hbar=\frac{c^{2}}{8.52 \times 10^{50}}
$$

This does not seem to give any deeper intuition before we replace $\hbar$ in the mass equation with this expression, which gives

$$
m=\frac{\hbar}{\bar{\lambda}} \frac{1}{c}=\frac{\frac{c^{2}}{8.52 \times 10^{50}}}{\bar{\lambda}} \frac{1}{c}=\frac{\frac{c}{\lambda}}{8.52 \times 10^{50}}
$$

Based on this, we will claim that the particle mass expressed in terms of $\mathrm{kg}$ is, at a deeper level, the reduced Compton frequency of that particle divided by the reduced Compton frequency of one $\mathrm{kg}$. For example, the reduced Compton frequency of an electron is

$$
f_{C}=\frac{c}{\bar{\lambda}_{e}} \approx 7.76 \times 10^{20}
$$

This means that the mass of the electron in terms of $\mathrm{kg}$ is

$$
m_{e}=\frac{\hbar}{\bar{\lambda}} \frac{1}{c}=\frac{f_{C, e}}{f_{C}} \approx \frac{7.76 \times 10^{20}}{8.52 \times 10^{50}} \approx 9.1 \times 10^{-31} \mathrm{~kg}
$$

This is the well-known mass of the electron. Still, keep in mind that the reduced Compton frequency of an electron is time dependent. If we have an observational time period of half a second, then the reduced Compton frequency is half of what it is in one second. However, the reduced Compton frequency of one $\mathrm{kg}$ will then be half of what we calculated above, so the ratio of the two will still be the same. That is, the mass in $\mathrm{kg}$ of an electron seems to be independent of the observational time-window.

If we should go to an observational time window shorter than the reduced Compton time $t_{C}=\frac{\bar{\lambda}}{c}$, then the reduced Compton frequency of the electron becomes less than one. We assume the reduced Compton frequency of matter must come in whole numbers and that a reduced Compton frequency of less than one should be treated as a probability, something we will return to later on. 
This reduced Compton frequency of one is very special. Interestingly, the reduced Compton frequency of the Planck mass in one Planck second is also one:

$$
\frac{c}{l_{p}} t_{p}=\frac{c}{l_{p}} \frac{l_{p}}{c}=1
$$

If the Planck time is the shortest possible time interval, then the Planck mass is the smallest mass, with a reduced Compton wavelength of one in the shortest possible time interval. The Planck mass is the very large compared to any observed particle, still we will see how this concept actually makes complete sense. For example, an electron is a Planck mass every Compton time, but the Planck mass only lasts one Planck second, so the mass of the electron is a fraction of a full mass per Compton time. In other words, the electron is much smaller than the Planck mass.

The idea that mass is related to Compton time has been supported by recent experimental research. Dolce and Perali [3] conclude that "the rest-mass of a particle is associated to a rest periodicity known as Compton periodicity". Also, [4] has concluded that "This directly demonstrates the connection between time and mass". In our view, the reduced Compton frequency hidden inside matter is the key to understanding matter more completely.

\section{A New and Simpler Definition of Mass}

The kg is simply an old mass measure. It likely came into existence to establish a standardized practical mass measure. What one knew about mass in those days was that one easily could measure the relative weight of mass and decided upon a clump of matter that was practical. One kg was not too heavy to carry around, neither was it too light. The history behind the $\mathrm{kg}$ becoming the mass standard is a long one, and beyond the scope of this paper. What is important is that we have pointed out that a mass, in terms of $\mathrm{kg}$, is simply the reduced Compton frequency ratio.

Again, the reduced Compton frequency of a Planck mass is one per Planck second. If this truly is essential, then a mass definition linked to the Planck mass rather than a $\mathrm{kg}$ will give us deeper insight and a series of new alternative equations in physics. We will suggest a new mass definition where the rest-mass is simply described as the reduced Compton frequency over one Planck second, that is

$$
\breve{m}=f_{C} t_{p}=\frac{c}{\bar{\lambda}} \frac{l_{p}}{c}=\frac{l_{p}}{\bar{\lambda}}
$$

we will use the notion $\breve{m}$ to distinguish this mass form from the standard mass form off $m$. This formula holds for any mass, from elementary particles to large composite masses. For all so called elementary particles, $\bar{\lambda} \geq l_{p}$, and if larger than $l_{p}$, then the reduced Compton frequency of that particle is less than one per Planck second. At a Planck second observational time window, the mass for Planck masses and below is actually the quantum probability for the particle to be in a Planck mass state. For a Planck mass particle, this probability is one, as $\bar{\lambda}=l_{p}$ and we then have $\breve{m}=\frac{l_{p}}{l_{p}}=1$. For all observed elementary particles, the mass is smaller than one. In our model, quantum probability and mass for elementary particles is the same. The mass of an electron is then simply

$$
\breve{m}_{e}=f_{C} t_{p}=\frac{c}{\bar{\lambda}_{e}} \frac{l_{p}}{c}=\frac{l_{p}}{\bar{\lambda}_{e}}=4.19 \times 10^{-23}
$$

That is the reduced Compton frequency of an electron over one Planck second. This is below one, and a frequency below one makes little sense, as we cannot observe a frequency below one. That is, it should be interpreted as a quantum probability for the electron to be in a mass Planck state in an observational time window of one Planck second. This means the electron is in a Planck mass state at every Compton time of the electron. However, this Planck mass state only lasts one Planck second. In a one-second observational time window, the electron is in a Planck mass state

$$
f_{C}=\frac{c}{\bar{\lambda}_{e}} \approx 7.76 \times 10^{20}
$$

times per second. However, each Planck mass state only lasts for one Planck second. So, this also is consistent with the $\mathrm{kg}$ definition of mass. That is, in terms of $\mathrm{kg}$, the electron has a mass of

$$
m_{e}=f_{C} m_{p} t_{p}=\frac{c}{\bar{\lambda}_{e}} \frac{\hbar}{l_{p}} \frac{l_{p}}{c}=\frac{\hbar}{\bar{\lambda}_{e}} \frac{1}{c}
$$

But back to our new mass formula, where $\breve{m}=\frac{l_{p}}{\lambda}$. For masses above the Planck mass, then one will end up with a number larger than one; the whole number part is something certain and the fractional part is a quantum probability. The mass of one $\mathrm{kg}$ in terms of reduced Compton frequency per second is

$$
\breve{m}_{k g}=\frac{l_{p}}{\bar{\lambda}}=\frac{l_{p}}{3.52 \times 10^{-43} \mathrm{~m}} \approx 45945119
$$


This is actual equal to the number of Planck masses in one $\mathrm{kg}$. So, if we multiply this number by the $\mathrm{kg}$ definition of one Planck mass, we get one kg. What is important is that our mass formula contains the Planck length, and does not contains the Planck constant.

With this mass definition, mass and energy are indistinguishable - both are reduced Compton frequencies. For elementary particles, that is particles with mass equal or below the Planck mass, energy and probability are the same thing. Actually at the deepest level, there only exist the Planck mass particle and light. The Planck mass particle is two colliding light particles.

Under our new mass definition, there is actually no mathematical difference between mass and energy. This simply indicates that at the Planck scale we cannot distinguish between mass and energy. The $c^{2}$ factor used to mathematically convert mass to energy is, in our view, a conversion factor that has more to do with choices of mass and energy definitions than with the deeper aspects of energy and mass. However, it is first when we understand mass can be seen as the reduced Compton frequency over a given time period that we easily can define mass and energy as indistinguishable at the Planck scale. We can easily extend our mass measure so that the $c^{2}$ factor is needed to convert mass to energy or energy to mass. An alternative and fully valid mass definition is what we can can call time-speed, defined as

$$
\bar{m}=\frac{l_{p}}{\bar{\lambda}} \frac{1}{c}
$$

That is the reduced Compton frequency per Planck second multiplied with one divided by the speed of light. In the special case of a Planck mass particle, we have $\bar{\lambda}=l_{p}$, which gives

$$
\bar{m}_{p}=\frac{l_{p}}{l_{p}} \frac{1}{c}=\frac{1}{c} \approx 3 \text { nano seconds per meter }
$$

That is a Planck mass particle is three nanoseconds per meter of light travelled. To convert this to energy, we multiply by $c^{2}$ and get

$$
\bar{E}=\bar{m} c^{2}=\frac{l_{p}}{\bar{\lambda}} c
$$

In the special case of a Planck mass energy, we have

$$
\bar{E}_{p}=\bar{m}_{p} c^{2}=\frac{l_{p}}{l_{p}} \frac{1}{c} c^{2}=c
$$

That is energy is related to speed, that is meters per second, while mass is seconds per meter. Does this mean we can come up with a long series of mass definitions? Yes and no. The mass and energy definitions must follow the relativistic "rules." The reduced Compton wavelength is, in our view, the only component of mass that can undergo length contraction. The Planck constant $\hbar$, or the speed of light $c$, or the Planck length are not affected by length contraction, something we will get back to soon. Both the standard mass measure and our two newly introduced mass definitions contains the reduced Compton wavelength of matter. However, the standard mass measure does not contain the Planck length; we think this is a mistake, as this is related to the particle aspect of the so-called "particle wave nature" of matter.

We think it best to select the simplest of these mass definitions that will help us simplify a series of formulas. As we will see, our definition of mass as simply reduced Compton frequency per Planck second seems to be the simplest formula that gets rid of both the Planck constant and the gravitational constant. Our second mass definition of time-speed leads to more familiar formulas and still we do not need $\hbar$ or $G$. Finally, the standard mass measure is the least simple one, it needs $\hbar$ because it links all mass to an old fashioned "artificial" (arbitrary) mass measure. This will become clear shortly.

\section{Relativistic Mass, Energy, and Probability}

Our new mass definition follows the Einstein [5] relativistic mass; that is, we have

$$
\begin{gathered}
\breve{m}=\frac{\breve{m}_{o}}{\sqrt{1-\frac{v^{2}}{c^{2}}}} \\
\breve{m}=\frac{\frac{l_{p}}{\lambda}}{\sqrt{1-\frac{v^{2}}{c^{2}}}} \\
\breve{m}=\frac{l_{p}}{\bar{\lambda} \sqrt{1-\frac{v^{2}}{c^{2}}}}
\end{gathered}
$$


We will see this equation is essential and we could even say "beautiful". We will claim no mass can have a relativistic reduced Compton frequency per Planck second of larger than one. This means we have a maximum relativistic mass equal to the Planck mass

$$
\begin{gathered}
\frac{l_{p}}{l_{p}}=\frac{l_{p}}{\bar{\lambda} \sqrt{1-\frac{v^{2}}{c^{2}}}} \\
1=\frac{l_{p}}{\bar{\lambda} \sqrt{1-\frac{v^{2}}{c^{2}}}}
\end{gathered}
$$

solving with respect to $v$, we get

$$
\begin{gathered}
\sqrt{1-\frac{v^{2}}{c^{2}}}=\frac{l_{p}}{\bar{\lambda}} \\
1-\frac{v^{2}}{c^{2}}=\frac{l_{p}^{2}}{\bar{\lambda}^{2}} \\
\frac{v^{2}}{c^{2}}=1-\frac{l_{p}^{2}}{\bar{\lambda}^{2}} \\
\frac{v}{c}=\sqrt{1-\frac{l_{p}^{2}}{\bar{\lambda}^{2}}} \\
v=\sqrt{1-\frac{l_{p}^{2}}{\bar{\lambda}^{2}}}
\end{gathered}
$$

Our assumptions lead to a maximum velocity for any elementary particle that is a function of the Planck length and the reduced Compton wavelength. The same maximum velocity of matter had been published by Haug [6-10]. It can also be derived using the standard mass definition by assuming the maximum relativistic mass is the Planck mass (as we do here), or by assuming the maximum length contraction a reduced Compton wavelength can undergo is such that it becomes the Planck length. Numerical calculations show this maximum velocity for an observed elementary particle is far above what can be achieved in current particle accelerators, but, as seen from the formula, clearly beyond the speed of light. Still, this maximum velocity may play a central role, in particular when it comes to the probabilistic interpretation of particles below the Planck mass size.

Based on Einstein-Poincare synchronized clocks, we basically get the same results as SR and kg measures, but the mathematics is simpler and more elegant when using our new mass measure, as shown in Table 1 . When we say "the same results," we mean relative to each other. We also see that a large number of calculations, including for length contraction, are independent of the mass definition, at least until we reach the Planck scale.

Table 2 shows the same as Table 1, but now with the elementary particle traveling at its maximum velocity speed $v_{\max }=c \sqrt{1-\frac{l_{p}^{2}}{\lambda^{2}}}$. As we can see, our new mass definition of reduced Compton frequency per Planck second gives very simple results.

Table 3 shows formulas for the Planck mass particle. This particle has a maximum velocity of $v_{\max }=$ $c \sqrt{1-\frac{l_{p}^{2}}{l_{p}^{2}}}=0$. This means the rest-mass formulas are identical to the relativistic formulas for the Planck mass particle. This means the Planck mass particle, the Planck length, and the Planck time are the same as seen from any observer across reference frames. This sounds remarkable and some will likely say unrealistic or even absurd. But it makes sense if you think of the Planck mass particle as two colliding light particles. Even if light "always" moves at the speed of light, in the moment the particles collide with each other they are (create) a Planck mass particle that lasts for one Planck second, and at that very instant (Planck second) light stands still, as it then creates mass. The Planck mass particle is the other aspect of the photon. In our Compton model, there is actually only one particle, the Planck mass particle, that consists of two colliding light particles. In 1965 Markov [11] actually supposed that mass that the Planck mass is the upper limit for the masses of elementary particles, this is also the case in our model.

\section{Quantum Probabilities}

The probability for a subatomic particle to be in a Planck mass state during a Planck second observational time window is given by the same formula as the newly defined mass formula. That is, for a rest-mass particle it is given by

$$
P=\frac{l_{p}}{\bar{\lambda}}
$$




\begin{tabular}{|c|c|c|c|}
\hline & $\begin{array}{c}\text { Standard mass: } \\
\text { mass as Compton } \\
\text { frequency per Planck time }\end{array}$ & $\begin{array}{c}\text { Alternative mass 1: } \\
\text { Mass as } \\
\text { kg (or pound) }\end{array}$ & $\begin{array}{c}\text { Alternative mass 2: } \\
\text { Mass as } \\
\text { Time-speed }\end{array}$ \\
\hline Rest-Mass & $\breve{m}=\frac{l_{p}}{\lambda}$ & $m=\frac{\hbar}{\lambda} \frac{1}{c}$ & $\bar{m}=\frac{l_{p}}{\lambda} \frac{1}{c}$ \\
\hline Rest-Mass Energy & $\breve{E}=\frac{t_{p}}{\lambda}$ & $E=\frac{\hbar}{\lambda} c$ & $\bar{E}=\frac{i_{p}}{\lambda} c$ \\
\hline Momentum & $\breve{p}=\frac{l_{p}}{\lambda} v$ & $p=\frac{\hbar}{\lambda} \frac{v}{c}$ & $\bar{p}=\frac{l_{p}}{\lambda} \frac{v}{c}$ \\
\hline Kinetic Energy & $\breve{E}_{K}=\frac{1}{2} \frac{l_{p}}{\lambda} \frac{v^{2}}{c^{2}}$ & $E_{K}=\frac{1}{2} \frac{\hbar}{\lambda} \frac{1}{c} v^{2}$ & $\bar{E}_{K}=\frac{1}{2} \frac{l_{p}}{\lambda} \frac{1}{c} v^{2}$ \\
\hline Rest-Mass Quantum Probability & $\frac{l_{p}}{\lambda}$ & $\frac{l_{p}}{\lambda}$ & $\frac{l_{p}}{\lambda}$ \\
\hline \multicolumn{4}{|l|}{ Relativistic formulas: } \\
\hline Relativistic Mass & $\breve{m}=\frac{l_{p}}{\bar{\lambda} \sqrt{1-\frac{v^{2}}{c^{2}}}}$ & $m=\frac{\hbar}{\bar{\lambda} \sqrt{1-\frac{v^{2}}{c^{2}}}} \frac{1}{c}$ & $\bar{m}=\frac{l_{p}}{\bar{\lambda} \sqrt{1-\frac{v^{2}}{c^{2}}}} \frac{1}{c}$ \\
\hline Relativistic Energy & $\breve{E}=\frac{l_{p}}{\bar{\lambda} \sqrt{1-\frac{v^{2}}{c^{2}}}}$ & $m=\frac{\hbar c}{\bar{\lambda} \sqrt{1-\frac{v^{2}}{c^{2}}}}$ & $\bar{E}=\frac{l_{p} c}{\bar{\lambda} \sqrt{1-\frac{v^{2}}{c^{2}}}}$ \\
\hline Kinetic Energy & $\breve{E}_{k}=\frac{l_{p}}{\bar{\lambda} \sqrt{1-\frac{v^{2}}{c^{2}}}}-\frac{l_{p}}{\bar{\lambda}}$ & $m=\frac{\hbar c}{\bar{\lambda} \sqrt{1-\frac{v^{2}}{c^{2}}}}-\frac{\hbar}{\lambda} \frac{1}{c}$ & $\bar{E}=\frac{l_{p} c}{\bar{\lambda} \sqrt{1-\frac{v^{2}}{c^{2}}}}-\frac{l_{p}}{\bar{\lambda}} \frac{1}{c}$ \\
\hline Relativistic Momentum & $p=\frac{l_{p} v}{\bar{\lambda} \sqrt{1-\frac{v^{2}}{c^{2}}}}$ & $p=\frac{\hbar}{\bar{\lambda} \sqrt{1-\frac{v^{2}}{c^{2}}}} \frac{v}{c}$ & $p=\frac{l_{p} v}{\bar{\lambda} \sqrt{1-\frac{v^{2}}{c^{2}}}} \frac{v}{c}$ \\
\hline Relativistic Quantum Probability & $\frac{l_{p}}{\bar{\lambda} \sqrt{1-\frac{v^{2}}{c^{2}}}}$ & $\frac{l_{p}}{\bar{\lambda} \sqrt{1-\frac{v^{2}}{c^{2}}}}$ & $\frac{l_{p}}{\bar{\lambda} \sqrt{1-\frac{v^{2}}{c^{2}}}}$ \\
\hline Length Contraction & $\bar{\lambda} \sqrt{1-\frac{v^{2}}{c^{2}}}$ & $\bar{\lambda} \sqrt{1-\frac{v^{2}}{c^{2}}}$ & $\bar{\lambda} \sqrt{1-\frac{v^{2}}{c^{2}}}$ \\
\hline Time Dilation & $\frac{\frac{\lambda}{c}}{\sqrt{1-\frac{v^{2}}{c^{2}}}}$ & $\frac{\frac{\lambda}{c}}{\sqrt{1-\frac{v^{2}}{c^{2}}}}$ & $\frac{\frac{\lambda}{c}}{\sqrt{1-\frac{v^{2}}{c^{2}}}}$ \\
\hline
\end{tabular}

Table 1: The table shows non-relativistic as well as relativistic formulas for elementary particles in physics, both with our new definition of mass and the standard $\mathrm{kg}$ definition of mass. The new definition of mass gives much simpler equations, and does so, we will claim, without losing out on anything important. As we will see when it comes to gravity, we have actually gained something important by using this mass definition, namely that we do not need Newton's gravitational constant or the Planck constant.

This mean for the Planck mass particle, the probability is always one, since the reduced Compton wavelength for a Planck particle is equal to the Planck length

$$
P=\frac{l_{p}}{l_{p}}=1
$$

For moving particles, we have that the probability for the particle to be in a Planck mass state during a given Planck second will be

$$
P=\frac{l_{p}}{\bar{\lambda} \sqrt{1-\frac{v^{2}}{c^{2}}}}
$$

At first glance, it seems like this probability can go above unity, if only $v$ is close enough to $c$. However, this is not the case when we take into account the maximum velocity for anything with rest-mass introduced in the section above. In that case, we have

$$
\begin{aligned}
P & =\frac{l_{p}}{\bar{\lambda} \sqrt{1-\frac{v_{\max }^{2}}{c^{2}}}} \\
P & =\frac{\overline{l_{p}}}{\bar{\lambda} \sqrt{1-\frac{\left(c \sqrt{1-\frac{l_{p}^{2}}{\bar{\lambda}^{2}}}\right)^{2}}{c^{2}}}} \\
P & =\frac{l_{p}}{\bar{\lambda} \sqrt{1-\frac{c^{2}\left(1-\frac{l_{p}^{2}}{\lambda^{2}}\right)}{c^{2}}}} \\
P & =\frac{l_{p}}{\bar{\lambda} \frac{l_{p}}{\lambda}} \\
P & =1
\end{aligned}
$$




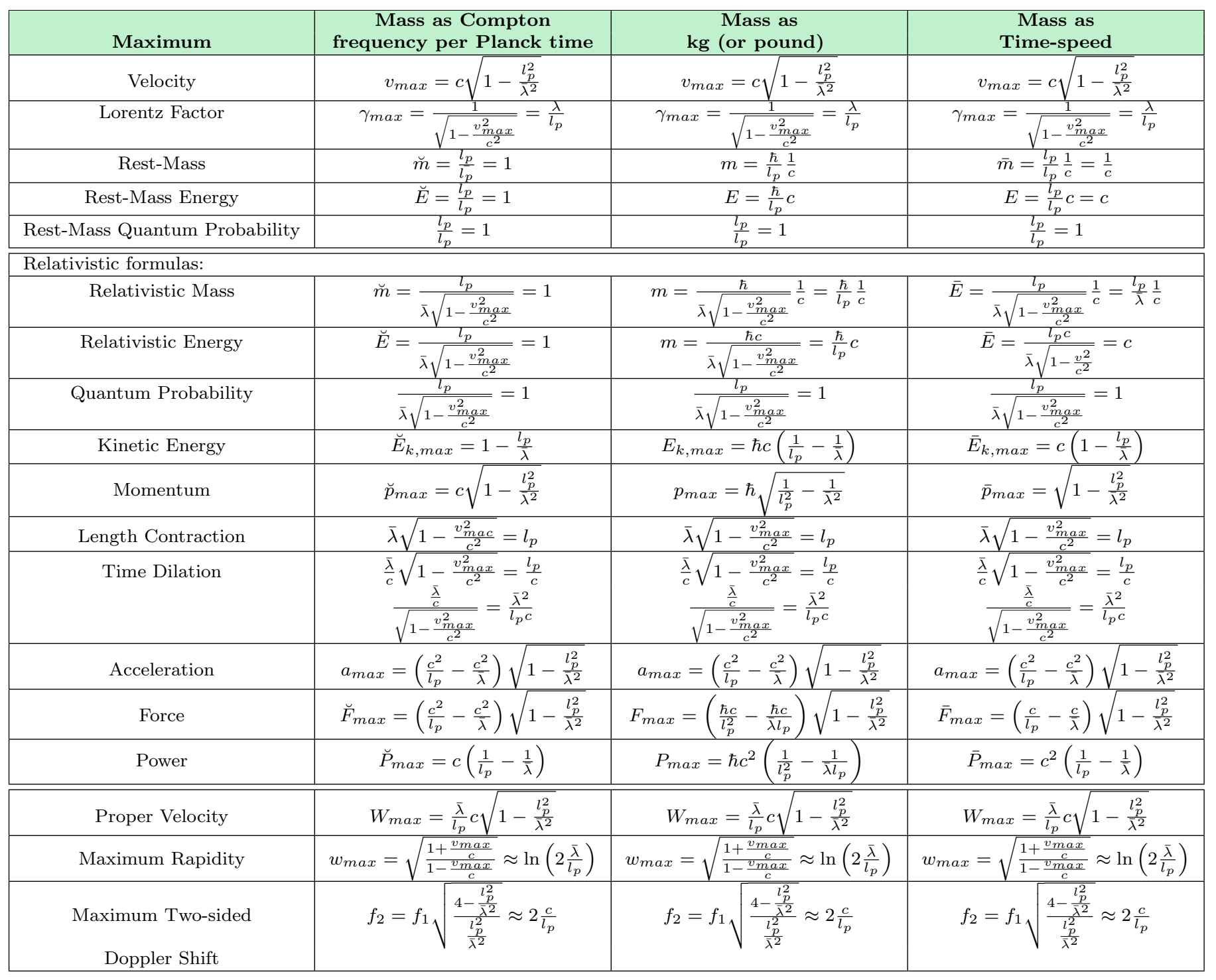

Table 2: The table shows non-relativistic as well as relativistic formulas for elementary particles in physics, both with our new definition of mass and the standard kg definition of mass just at the moment it travels at its maximum velocity. Pay particular attention to the fact that the quantum probabilities are one in this case. In other words, uncertainty disappears at Planck scale energies.

That is, the probability of finding an elementary particle in its Planck mass state at the moment it reaches its maximum velocity is one. Also, the Planck mass state, energy, and quantum probability are not distinguishable when we use our definition of mass where mass is reduced Compton frequency per Planck second. If we use mass as kg instead then mass, energy, and quantum probability are all described with different formulas, which is to say, mass, energy, and probability are then not easy to see as the same thing at a deeper level. Still, the quantum probability for the particle being in the Planck mass state is always given by this formula.

Haug [12] has recently claimed to have shown that this leads to a collapse of Heisenberg's uncertainty principle at the Planck scale. This holds no matter what the mass definition is, but it is not as dramatic as it sounds because the breakdown of uncertainty only lasts inside one Planck second. An analogy would be a binary system that flipped every Planck second. But if you watched for less than one Planck second, then it could not flip inside that Planck second. A probability of $100 \%$ in relation to observing the Planck mass particle inside of one Planck second (based on one knowing it is a Planck mass particle) is 100\%. We claim the particle wave duality collapses at the Planck particle. Either the Planck particle exists and then it lasts for one Planck second, or it has dissolved into light. 


\begin{tabular}{|c|c|c|c|}
\hline $\begin{array}{c}\text { Planck Mass Particle } \\
\text { Maximum }\end{array}$ & $\begin{array}{c}\text { Mass as Compton } \\
\text { frequency per Planck time }\end{array}$ & $\begin{array}{l}\text { Mass as } \\
\mathrm{kg} \text { (or pound) }\end{array}$ & $\begin{array}{c}\text { Mass as } \\
\text { Time-speed }\end{array}$ \\
\hline Rest-Mass & $\breve{m}=\frac{l_{p}}{l_{p}}=1$ & $m=\frac{\hbar}{l_{p}} \frac{1}{c}$ & $\bar{m}=\frac{l_{p}}{l_{p}} \frac{1}{c}=\frac{1}{c}$ \\
\hline Rest-Mass Energy & $\breve{E}=\frac{l_{p}}{l_{p}}=1$ & $E=\frac{\hbar}{l_{p}} c$ & $\bar{E}=\frac{l_{p}}{l_{p}} c=c$ \\
\hline Velocity & $v_{\max }=c \sqrt{1-\frac{l_{p}^{2}}{l_{p}^{2}}}=0$ & $v_{\max }=c \sqrt{1-\frac{l_{p}^{2}}{l_{p}^{2}}}=0$ & $v_{\max }=c \sqrt{1-\frac{l_{p}^{2}}{l_{p}^{2}}}=0$ \\
\hline Lorentz Factor & $\gamma_{\max }=\frac{1}{\sqrt{1-\frac{v_{\max }^{2}}{c^{2}}}}=1$ & $\gamma_{\max }=\frac{1}{\sqrt{1-\frac{v_{\max }^{2}}{c^{2}}}}=1$ & $\gamma_{\max }=\frac{1}{\sqrt{1-\frac{v_{\max }^{2}}{c^{2}}}}=1$ \\
\hline Rest-Mass Quantum Probability & $\frac{l_{p}}{l_{p}}=1$ & $\frac{l_{p}}{l_{p}}=1$ & $\frac{l_{p}}{l_{p}}=1$ \\
\hline \multicolumn{4}{|l|}{ Relativistic formulas: } \\
\hline Relativistic Mass & $\breve{m}=\frac{l_{p}}{l_{p} \sqrt{1-\frac{v_{\max }^{2}}{c^{2}}}}=1$ & $m=\frac{\hbar}{l_{p} \sqrt{1-\frac{v_{\max }^{2}}{c^{2}}}} \frac{1}{c}=\frac{\hbar}{l_{p}} \frac{1}{c}$ & $\bar{E}=\frac{l_{p}}{l_{p} \sqrt{1-\frac{v_{\max }^{2}}{c^{2}}}} \frac{1}{c}=\frac{l_{p}}{\lambda} \frac{1}{c}$ \\
\hline Relativistic Energy & $\breve{E}=\frac{l_{p}}{l_{p} \sqrt{1-\frac{v_{\max }^{2}}{c^{2}}}}=1$ & $m=\frac{\hbar c}{\bar{\lambda} \sqrt{1-\frac{v_{\max }^{2}}{c^{2}}}}=\frac{\hbar}{l_{p}} c$ & $\bar{E}=\frac{l_{p} c}{\bar{\lambda} \sqrt{1-\frac{v^{2}}{c^{2}}}}=c$ \\
\hline Quantum Probability & $\frac{l_{p}}{l_{p} \sqrt{1-\frac{v_{\max }^{2}}{c^{2}}}}=1$ & $\frac{l_{p}}{\bar{\lambda} \sqrt{1-\frac{v_{\max }^{2}}{c^{2}}}}=1$ & $\frac{l_{p}}{\bar{\lambda} \sqrt{1-\frac{v_{\max }^{2}}{c^{2}}}}=1$ \\
\hline Kinetic Energy & $\breve{E}_{k, \max }=1-\frac{l_{p}}{l_{p}}=0$ & $E_{k, \max }=\hbar c\left(\frac{1}{l_{p}}-\frac{1}{l_{p}}\right)=0$ & $\bar{E}_{k, \max }=c\left(1-\frac{l_{p}}{l_{p}}\right)=0$ \\
\hline Momentum & $\breve{p}_{\max }=c \sqrt{1-\frac{l_{p}^{2}}{l_{p}^{2}}}=0$ & $p_{\max }=\hbar \sqrt{\frac{1}{l_{p}^{2}}-\frac{1}{l_{p}^{2}}}=0$ & $\bar{p}_{\max }=\sqrt{1-\frac{l_{p}^{2}}{l_{p}^{2}}}=0$ \\
\hline Length Contraction & $l_{p} \sqrt{1-\frac{v_{m a c}^{2}}{c^{2}}}=l_{p}$ & $\bar{\lambda} \sqrt{1-\frac{v_{m a x}^{2}}{c^{2}}}=l_{p}$ & $\bar{\lambda} \sqrt{1-\frac{v_{\max }^{2}}{c^{2}}}=l_{p}$ \\
\hline Time Dilation & $\begin{array}{c}\frac{l_{p}}{c} \sqrt{1-\frac{v_{\max }^{2}}{c^{2}}}=\frac{l_{p}}{c} \\
\frac{\frac{l_{p}}{c}}{\sqrt{1-\frac{v_{\max }^{2}}{c^{2}}}}=\frac{l_{p}}{c}\end{array}$ & $\begin{array}{c}\frac{l_{p}}{c} \sqrt{1-\frac{v_{\max }^{2}}{c^{2}}}=\frac{l_{p}}{c} \\
\frac{\frac{l_{p}}{c}}{\sqrt{1-\frac{v_{\max }^{2}}{c^{2}}}}=\frac{l_{p}}{c}\end{array}$ & $\begin{array}{c}\frac{l_{p}}{c} \sqrt{1-\frac{v_{\max }^{2}}{c^{2}}}=\frac{l_{p}}{c} \\
\frac{\frac{l_{p}}{c}}{\sqrt{1-\frac{v_{\max }^{2}}{c^{2}}}}=\frac{l_{p}}{c}\end{array}$ \\
\hline Acceleration & $a_{\max }=\left(\frac{c^{2}}{l_{p}}-\frac{c^{2}}{l_{p}}\right) \sqrt{1-\frac{l_{p}^{2}}{l_{p}^{2}}}=0$ & $a_{\max }=\left(\frac{c^{2}}{l_{p}}-\frac{c^{2}}{l_{p}}\right) \sqrt{1-\frac{l_{p}^{2}}{l_{p}^{2}}}=0$ & $a_{\max }=\left(\frac{c^{2}}{l_{p}}-\frac{c^{2}}{l_{p}}\right) \sqrt{1-\frac{l_{p}^{2}}{l_{p}^{2}}}=0$ \\
\hline Force & $\breve{F}_{\max }=\left(\frac{c^{2}}{l_{p}}-\frac{c^{2}}{l_{p}}\right) \sqrt{1-\frac{l_{p}^{2}}{\lambda^{2}}}=0$ & $F_{\max }=\left(\frac{\hbar c}{l_{p}^{2}}-\frac{\hbar c}{l_{p}^{2}}\right) \sqrt{1-\frac{l_{p}^{2}}{l_{p}^{2}}}$ & $\bar{F}_{\max }=\left(\frac{c}{l_{p}}-\frac{c}{l_{p}}\right) \sqrt{1-\frac{l_{p}^{2}}{l_{p}^{2}}}=0$ \\
\hline Rest Force & $\breve{F}_{\max }=\frac{c^{2}}{l_{p}}$ & $F_{\max }=\frac{\hbar c}{l_{p}^{2}}$ & $\bar{F}_{\max }=\frac{c}{l_{p}}$ \\
\hline Power & $\breve{P}_{\text {max }}=c\left(\frac{1}{l_{p}}-\frac{1}{l_{p}}\right)=0$ & $P_{\max }=\hbar c^{2}\left(\frac{1}{l_{p}^{2}}-\frac{1}{l_{p}^{2}}\right)=0$ & $\bar{P}_{\max }=c^{2}\left(\frac{1}{l_{p}}-\frac{1}{l_{p}}\right)=0$ \\
\hline Rest Power & $\breve{P}_{\max }=\frac{c}{l_{p}}$ & $P_{\max }=\frac{\hbar c^{2}}{l_{p}^{2}}=0$ & $\bar{P}_{\max }=\frac{c^{2}}{l_{p}}$ \\
\hline Proper Velocity & $W_{\max }=\frac{\bar{\lambda}}{l_{p}} c \sqrt{1-\frac{l_{p}^{2}}{l_{p}^{2}}}=0$ & $W_{\max }=\frac{\bar{\lambda}}{l_{p}} c \sqrt{1-\frac{l_{p}^{2}}{l_{p}^{2}}}=0$ & $W_{\max }=\frac{\bar{\lambda}}{l_{p}} c \sqrt{1-\frac{l_{p}^{2}}{l_{p}^{2}}}=0$ \\
\hline Maximum Rapidity & $w_{\max }=\sqrt{\frac{1+\frac{v_{\max }}{c}}{1-\frac{v_{\max }}{c}}}=1$ & $w_{\max }=\sqrt{\frac{1+\frac{v_{\max }}{c}}{1-\frac{v_{\max }}{c}}}=1$ & $w_{\max }=\sqrt{\frac{1+\frac{v_{\max }}{c}}{1-\frac{v_{\max }}{c}}}=1$ \\
\hline $\begin{array}{c}\text { Maximum Two-sided } \\
\text { Doppler Shift }\end{array}$ & $f_{2}=f_{1} \sqrt{\frac{1+\frac{v_{\max }}{c}}{1-\frac{v_{\max }}{c}}}=f_{1}$ & $f_{2}=f_{1} \sqrt{\frac{1+\frac{v_{\max }}{c}}{1-\frac{v_{\max }}{c}}}=f_{1}$ & $f_{2}=f_{1} \sqrt{\frac{1+\frac{v_{\max }}{c}}{1-\frac{v_{\max }}{c}}}=f_{1}$ \\
\hline
\end{tabular}

Table 3: The table shows non-relativistic as well as relativistic formulas for a series of well-known entities in physics, both with our new definition of mass and the standard $\mathrm{kg}$ definition of mass

\section{Relativistic Energy Momentum Relation}

The relativistic energy momentum relation plays a central role in modern physics, as it also is used in quantum physics. Under modern physics, it is given by $E^{2}=p^{2} c^{2}+m c^{2}$. However, this is based on a special definition of mass as well as energy, namely mass in terms of $\mathrm{kg}$ (weight). When we define mass and energy as reduced Compton frequency per Planck second, then the relativistic energy momentum relation takes the simpler form of

$$
E^{2}=\frac{p^{2}}{c^{2}}+m^{2}
$$

This gives 


$$
\begin{aligned}
& E=\sqrt{\frac{p^{2}}{c^{2}}+m^{2}} \\
& E=\sqrt{\left(\frac{\frac{l_{p}}{\lambda} v}{\sqrt{1-\frac{v^{2}}{c^{2}}}}\right)^{2} \frac{1}{c^{2}}+\left(\frac{l_{p}}{\bar{\lambda}}\right)^{2}} \\
& E=\sqrt{\frac{\frac{l_{p}^{2}}{\bar{\lambda}^{2}} \frac{v^{2}}{c^{2}}}{1-\frac{v^{2}}{c^{2}}}+\frac{l_{p}^{2}}{\bar{\lambda}^{2}}} \\
& E=\sqrt{\frac{\frac{l_{p}^{2}}{\lambda^{2}}\left(\frac{v^{2}}{c^{2}}-1\right)}{1-\frac{v^{2}}{c^{2}}}+\frac{\frac{l_{p}^{2}}{\lambda^{2}}}{1-\frac{v^{2}}{c^{2}}}+\frac{l_{p}^{2}}{\bar{\lambda}^{2}}} \\
& E=\sqrt{-\frac{l_{p}^{2}}{\bar{\lambda}^{2}}+\frac{\frac{l_{p}^{2}}{\bar{\lambda}^{2}}}{1-\frac{v^{2}}{c^{2}}}+\frac{l_{p}^{2}}{\bar{\lambda}^{2}}} \\
& E=\sqrt{\frac{\frac{l_{p}^{2}}{\lambda^{2}}}{1-\frac{v^{2}}{c^{2}}}} \\
& E=\frac{\frac{l_{p}}{\lambda}}{\sqrt{1-\frac{v^{2}}{c^{2}}}} \\
& E=\frac{l_{p}}{\bar{\lambda} \sqrt{1-\frac{v^{2}}{c^{2}}}}
\end{aligned}
$$

That is, we end up with the expression for relativistic energy as we had earlier. When utilizing our maximum velocity formula, we will claim the same relativistic energy momentum relation also holds for light. Light has mass, in our view, at the very moment (one Planck second) that two light particles collide. They then have (or we should say create) the mass gap that is equal to the Planck mass over one Planck second, which is 1 under the mass definition above and $\frac{\hbar}{c^{2}}$ in the $\mathrm{kg}$ definition. Just for comparison, we show the relativistic energy momentum relation when mass is under the standard $\mathrm{kg}$ definition:

$$
\begin{aligned}
& E=\sqrt{p^{2} c^{2}+\left(m c^{2}\right)^{2}} \\
& E=\sqrt{\left(\frac{m v}{\sqrt{1-\frac{v^{2}}{c^{2}}}}\right)^{2} c^{2}+\left(m c^{2}\right)^{2}} \\
& E=\sqrt{\frac{m^{2} v^{2} c^{2}}{1-\frac{v_{e}^{2}}{c^{2}}}+m_{t}^{2} c^{4}} \\
& E=\sqrt{\frac{m^{2} \frac{v^{2}}{c^{2}} c^{4}}{1-\frac{v^{2}}{c^{2}}}+m^{2} c^{4}} \\
& E=\sqrt{\frac{m^{2} c^{4}\left(\frac{v^{2}}{c^{2}}-1\right)}{1-\frac{v^{2}}{c^{2}}}+\frac{m^{2} c^{4}}{1-\frac{v^{2}}{c^{2}}}+m^{2} c^{4}} \\
& E=\sqrt{-m^{2} c^{4}+\frac{m^{2} c^{4}}{1-\frac{v^{2}}{c^{2}}}+m^{2} c^{4}} \\
& E=\sqrt{\frac{m^{2} c^{4}}{1-\frac{v^{2}}{c^{2}}}} \\
& E=\frac{m c^{2}}{\sqrt{1-\frac{v^{2}}{c^{2}}}} \\
& E=\frac{\hbar}{\bar{\lambda} \sqrt{1-\frac{v^{2}}{c^{2}}}} c
\end{aligned}
$$


This latter equation gives less intuition, as it contains the Planck constant. However, it is simply the reduced Compton frequency of the elementary particle divided by the reduced Compton frequency in one kg. The reduced Compton frequency in the particle is adjusted for relativistic effects.

\section{A Series of Different Values for $G$ and even Different Gravi- tational Force Formulas Gives the Same Gravity Predictions}

The truly great insight from Newton concerns the inverse square law of the gravitational force with respect to distance [13]. Newton himself never used the gravitational constant or found its value. It was first found empirically and indirectly by Cavendish in 1798 using a so-called Cavendish apparatus [14].

Next, we will show three formulas similar to Newton's universal gravitational formula, but all with a different gravitational constant that is dependent on the mass definition. All of them predict the same observable results in relation to gravity. The Newton gravitational constant, we will see, is directly linked to one specific way of defining mass, namely as $\mathrm{kg}$ or pound. There is nothing wrong with that, but there are other ways to look at and measure mass that are simpler and more intuitive. Only the standard mass measure that is related to Newton's gravitational constant must rely on the Planck constant to find the Planck length. This is because the Planck constant is actually embedded in the Newton constant. The essential elements for gravity are the Planck length and the speed of light, which is equal to the speed of gravity. The Newton gravitational constant, as well as the Planck constant, are not really needed for any calculations in physics. That is, when we switch mass measure $G$ then $\hbar$ is never needed.

The Newton universal gravitational formula is given by

$$
F=G \frac{m M}{R^{2}}
$$

However, we claim that the Newton gravitational constant is a composite constant of the form (see [6, 15, 16])

$$
G=\frac{l_{p}^{2} c^{3}}{\hbar}
$$

This means we can rewrite the Newton gravitational formula as

$$
F=G \frac{\bar{m} \bar{M}}{R^{2}}=G=\frac{l_{p}^{2} c^{3}}{\hbar} \frac{\frac{\hbar}{\lambda_{M}} \frac{1}{c} \frac{\hbar}{\lambda_{m}} \frac{1}{c}}{R^{2}}=\frac{\hbar c}{R^{2}} \frac{l_{p}}{\bar{\lambda}_{M}} \frac{l_{p}}{\bar{\lambda}_{m}}
$$

Alternatively, we can define mass as only the reduced Compton frequency of the mass in question over one Planck second.

$$
F=\breve{G} \frac{\breve{m} \breve{M}}{R^{2}}=l_{p} c^{2} \frac{\frac{l_{p}}{\lambda_{M}} \frac{l_{p}}{\lambda_{m}}}{R^{2}}=\frac{l_{p} c^{2}}{R^{2}} \frac{l_{p}}{\bar{\lambda}_{M}} \frac{l_{p}}{\bar{\lambda}_{m}}
$$

That is to say, we now claim under this alternative mass measure that the gravitational constant now is $\breve{G}=l_{p} c^{2}$, which is very different than the Newton gravitational constant. .

A third alternative is to define the mass equal to as

$$
\bar{m}=f_{C} t_{p} \frac{1}{c}=\frac{l_{p}}{\bar{\lambda}} \frac{1}{c}
$$

this gives

$$
F=\bar{G} \frac{\bar{m} \bar{M}}{R^{2}}=l_{p} c^{3} \frac{\frac{l_{p}}{\lambda_{M}} \frac{1}{c} \frac{l_{p}}{\lambda_{m}} \frac{1}{c}}{R^{2}}=\frac{l_{p} c}{R^{2}} \frac{l_{p}}{\bar{\lambda}_{M}} \frac{l_{p}}{\bar{\lambda}_{m}}
$$

where $\bar{G}=l_{p} c^{3}$. Even if all these gravitational force formulas are different and their gravitational constants are different, they all give exactly the same gravitational predictions. That is, the Newton gravitational constant is not even just a composite constant, it is not a unique gravity formulation.

What is important is that for all gravity phenomena we need the Compton frequency of the matter relative to the Planck frequency and also the speed of light. The Newton gravitational constant contains embedded the Planck constant in addition. This gets rid of the Planck constant embedded in the standard mass definition, so one can convert the mass into what is important for gravity.

In macroscopic masses, $\bar{\lambda}$ is considerably smaller than $l_{p}$ and $\frac{l_{p}}{\lambda}$ will then be equal to an integer number that tells how many Planck masses in the mass we are working with. Still, behind this number is the Compton frequency of the mass in question divided by the Compton frequency of the Planck mass. So, to find this we need to know both the Compton length of the mass in question and the Planck length; both can be found independent of Newton's gravitational constant. 


\begin{tabular}{|c|c|c|c|}
\hline & Newton & Alternative-1 & Alternative-2 \\
\hline Mass seen as & $\begin{array}{l}\text { Compton frequency } \\
\text { relative to Compton frequency kg }\end{array}$ & $\begin{array}{l}\text { Reduced Compton frequency } \\
\text { in one Planck second }\end{array}$ & $\begin{array}{l}\text { Reduced Compton frequency } \\
\text { in one Planck second } \times \frac{1}{c}\end{array}$ \\
\hline Mass mathematically & $M=\frac{\hbar}{\lambda} \frac{1}{c}$ & $\breve{M}=\frac{l_{p}}{\lambda}$ & $\bar{M}=\frac{l_{p}}{\lambda} \frac{1}{c}$ \\
\hline Gravity constant & $G=\frac{l_{p}^{2} c^{3}}{\hbar}$ & $\breve{G}=l_{p} c^{2}$ & $\bar{G}=l_{p} c^{3}$ \\
\hline \multicolumn{4}{|c|}{ Non "observable" predictions: } \\
\hline Gravity force & $F=G \frac{M m}{R^{2}}$ & $\breve{F}=\breve{G} \frac{M \breve{m}}{R^{2}}$ & $\bar{F}=\bar{G} \frac{M \bar{m}}{R^{2}}$ \\
\hline Gravity force & $F=\frac{\hbar c}{R^{2}} \frac{l_{p}}{\lambda} \frac{l_{p}}{\lambda}$ & $\breve{F}=\frac{l_{p} c^{2}}{R^{2}} \frac{l_{p}}{\lambda} \frac{l_{p}}{\lambda}$ & $\bar{F}=\frac{l_{p} c^{2}}{R^{2}} \frac{l_{p}}{\lambda} \frac{l_{p}}{\lambda}$ \\
\hline \multicolumn{4}{|l|}{ Observable predictions: } \\
\hline Gravity acceleration & $g=c^{2} \frac{l_{p}}{R^{2}} \frac{l_{p}}{\lambda}$ & $g=c^{2} \frac{l_{p}}{R^{2}} \frac{l_{p}}{\lambda}$ & $g=c^{2} \frac{l_{p}}{R^{2}} \frac{l_{p}}{\lambda}$ \\
\hline Orbital velocity & $v_{o}=c \sqrt{\frac{l_{p}}{R} \frac{l_{p}}{\lambda}}$ & $v_{o}=c \sqrt{\frac{l_{p}}{R} \frac{l_{p}}{\lambda}}$ & $v_{o}=c \sqrt{\frac{l_{p}}{R} \frac{l_{p}}{\lambda}}$ \\
\hline Escape velocity & $v_{e}=c \sqrt{2 \frac{l_{p}}{R} \frac{l_{p}}{\lambda}}$ & $v_{e}=c \sqrt{2 \frac{l_{p}}{R} \frac{l_{p}}{\lambda}}$ & $v_{e}=c \sqrt{2 \frac{l_{p}}{R} \frac{l_{p}}{\lambda}}$ \\
\hline Time dilation & $T_{R}=T_{f} \sqrt{1-\frac{v_{e}^{2}}{c^{2}}}$ & $T_{R}=T_{f} \sqrt{1-\frac{v_{e}^{2}}{c^{2}}}$ & $T_{R}=T_{f} \sqrt{1-\frac{v_{e}^{2}}{c^{2}}}$ \\
\hline Gravitational red-shift & $z(r)=\frac{l_{p}}{r} \frac{l_{p}}{\lambda}$ & $z(r)=\frac{l_{p}}{r} \frac{l_{p}}{\lambda}$ & $z(r)=\frac{l_{p}}{r} \frac{l_{p}}{\lambda}$ \\
\hline Schwarzschild radius & $r_{s}=l_{p} \frac{l_{p}}{\lambda}$ & $r_{s}=l_{p} \frac{l_{p}}{\lambda}$ & $r_{s}=l_{p} \frac{l_{p}}{\lambda}$ \\
\hline Energy & $E=M c^{2}$ & $E=M$ & $\bar{E}=M c^{2}$ \\
\hline
\end{tabular}

Table 4: The table shows the Newton gravitational force in addition to two alternative Newton-type theories, but with different gravitational constants that all predict the same.

It is also worth noting that if we define mass as reduced Compton frequency per Planck time, then mass and energy are actually identical. There is no $c^{2}$ needed between the two to transfer mass to energy. Also, the $c^{2}$ has to do with our definition of mass as $k g$, weight and our definition of energy. However, the reduced Compton frequency per Planck time as a mass definition does not mathematically distinguish mass from energy. It does not say anything about what this frequency is doing relative to an energy frequency. If we want to this approach, then we can choose our time-speed mass definition, and we need $c^{2}$ to convert to our energy measure.

\section{Finding the Plank Length without the Newtonian $G$ and without $\hbar$ from a Cavendish Apparatus}

Using a Cavendish apparatus, we can measure the Planck length without any knowledge of Newton's gravitational constant, or any knowledge of the Planck constant. A Cavendish apparatus consist of two small balls and two larger balls, all made of lead, for example. The torque (moment of force) is given by

$$
\kappa \theta
$$

where $\kappa$ is the torsion coefficient of the suspending wire and $\theta$ is the deflection angle of the balance. We then have the following well-known relationship

$$
\kappa \theta=L F
$$

where $L$ is the length between the two small balls in the apparatus. Further, $F$ can be set equal to Newton's formula, but with a Compton view of matter and therefore no need for Newton's gravitational constant, this is important to help us bypass the need for the Planck constant as well.

Our Newton-equivalent gravity formula is equal to

$$
\breve{F}=\frac{\breve{M} \breve{m}}{R^{2}}=l_{p} c^{2} \frac{\frac{l_{p}}{\lambda_{M}} \frac{l_{p}}{\lambda_{m}}}{R^{2}}
$$

where we assume the size of $l_{p}$ is unknown.

This means we must have

$$
\kappa \theta=L l_{p} c^{2} \frac{\breve{M} \breve{m}}{R^{2}}
$$

We also have that the natural resonant oscillation period of a torsion balance is given by 


$$
T=2 \pi \sqrt{\frac{I}{\kappa}}
$$

Further, the moment of inertia $I$ of the balance is given by

$$
I=m\left(\frac{L}{2}\right)^{2}+m\left(\frac{L}{2}\right)^{2}=2 m\left(\frac{L}{2}\right)^{2}=\frac{m L^{2}}{2}
$$

this means we have

$$
T=2 \pi \sqrt{\frac{m L^{2}}{2 \kappa}}
$$

and when solved with respect to $\kappa$, this gives

$$
\begin{aligned}
\frac{T^{2}}{2^{2} \pi^{2}} & =\frac{m L^{2}}{2 \kappa} \\
\kappa & =\frac{m L^{2}}{2 \frac{T^{2}}{2^{2} \pi^{2}}} \\
\kappa & =\frac{m L^{2} 2 \pi^{2}}{T^{2}}
\end{aligned}
$$

Next, in equation 40 we are replacing $\kappa$ with this expression

$$
\begin{aligned}
\frac{m L^{2} 2 \pi^{2}}{T^{2}} \theta & =L l_{p} c^{2} \frac{\breve{M} \breve{m}}{R^{2}} \\
\frac{L^{2} 2 \pi^{2}}{T^{2}} \theta & =L l_{p} c^{2} \frac{\breve{M}}{R^{2}}
\end{aligned}
$$

Next remember our mass definition is $\breve{M}=\frac{l_{p}}{\lambda}$, which we now replace in the equation above and solving with respect to the unknown Planck length, we get

$$
\begin{aligned}
\frac{L^{2} 2 \pi^{2}}{T^{2}} \theta & =L l_{p} c^{2} \frac{\frac{l_{p}}{\lambda}}{R^{2}} \\
\frac{L^{2} 2 \pi^{2}}{T^{2}} \theta & =L l_{p}^{2} \frac{\frac{c^{2}}{R^{2}}}{R^{2}} \\
\frac{L 2 \pi^{2} R^{2}}{T^{2} \frac{c^{2}}{\lambda}} \theta & =l_{p}^{2} \\
l_{p} & =\sqrt{\frac{L 2 \pi^{2} R^{2}}{T^{2} \frac{c^{2}}{\lambda}} \theta} \\
l_{p} & =\sqrt{\frac{L 2 \pi^{2} R^{2} \theta}{T^{2} f_{C} c}}
\end{aligned}
$$

where $f_{C}$ is the reduced Compton frequency of the mass in question. And again, the reduced Compton frequency can be found without $\hbar$ by first measuring the reduced Compton wavelength of the electron. Then calculate the reduced Compton frequency $f_{C, e}=\frac{c}{\lambda_{e}}$. Next run a Cyclotron experiment to find that the reduced Compton frequency of the electron is about 1,836 times that of the electron. For example, [17] measured the proton mass relative to the electron mass to be $1836.152470(76)$ using a Cylotron, but we need no knowledge of the mass in $\mathrm{kg}$ (weight), as we must have $\frac{f_{C, P}}{f_{C, e}}=\frac{m_{P}}{m_{e}}$, where $f_{C, e}$ is the reduced Compton frequency of the proton and $f_{C, e}$ is the reduced Compton frequency of the electron. Next pack a significant mass with protons (atoms where one knows number of protons). Based on this, one has the reduced Compton frequency of the composite mass that we are going to use in the Cavendish apparatus. Alternatively, one can use the Planck constant also as $\frac{c^{2}}{\hbar}$ is the reduced Compton frequency in one $\mathrm{kg}$, and if one then finds the large ball weight irrelative to one $\mathrm{kg}$.

We will claim that the reduced Compton wavelength together with the Planck length is what better defines the so-called wave-particle duality of matter. However, modern physics actually seems to have a incomplete information about the particle structure in its mass formulas, we find the Compton frequency embedded, but not the Planck length. The standard mass definition is then adjusted by using an empirically estimated gravitational constant that indirectly must capture the Planck mass (and thereby the Planck length) aspect of matter and is why, in our view, the Newton gravitational constant embedded contains the Planck length, $G=\frac{l_{p}^{2} c^{3}}{\hbar}$. 
It is also worth pointing out that the Schwarzschild-radius in a Cavendish apparatus is given by

$$
R_{s}=\frac{L 4 \pi^{2} R^{2} \theta}{T^{2} c^{2}}
$$

This means that the Planck length also is given by

$$
l_{p}=\sqrt{\frac{1}{2} \frac{r_{s}}{\bar{\lambda}}}
$$

That is, the Planck length is given by the square root of half the Schwarzschild radius divided by the reduced Compton matter of the gravity object (mass). What is important here is that we can find the Planck length independent of Newton's gravitational constant and the Planck constant. This because we also do not need to know $G$ or $\hbar$ to find the Schwarzschild radius or the reduced Compton wavelength.

\section{Why Newton's Gravitational Constant Clearly Is a Compos- ite Constant}

- Already when looking at the output units of Newton's gravitational constant $\mathrm{m}^{3} \cdot \mathrm{kg}^{-1} \cdot \mathrm{s}^{-2}$, one should suspect that this must be a composite constant. It is highly unlikely that the universe invented something fundamental that has some complex unities. Much more likely is that the Planck length is something very fundamental. Everyone can intuitively understand somewhat what a length is, one can also to a large degree understand what the speed of light is, it is how far the light travels for a given time period.

- The Planck length can be found totally independent of any knowledge of Newton's gravitational constant, and it can also be found independent of the Planck constant, as shown in this paper. Further, it is the Planck length that is needed in all observable gravitational predictions, see Table 4. The Planck constant in the Planck formula, is in our view able actually to cancel out of the Planck constant embedded in Newton's gravitational constant. The Newton gravitational constant is needed to get rid of the Planck constant, that is linked to the weight definition of matter.

- We have shown three more alternative Newton equivalent gravity theories that have different gravitational constant and different output and formulas at a deeper level. These formulas need less information, as they are calibrated to more fundamental aspects of matter. When mass is $\mathrm{kg}$, one can calibrate to this "artificial" mass measure.

- The Schwarzschild radius and every other predictable aspect of gravity can be found without Newton's gravitational constant. The gravity force itself is, to our knowledge, never observed directly, only other aspects of it.

- By reformulating $G$ as a composite of the form $G=\frac{l_{p}^{2} c^{3}}{\hbar}$, a long series of the Planck units are dramatically simplified and become more logical. The Planck time described as $t_{p}=\sqrt{\frac{G \hbar}{c^{5}}}$ gives minimal intuition. We may ask, what is the logical meaning of $c^{5}$ and what is the deeper logic behind the gravitational constant? First, when replacing $G$ with its composite form, we see that the Planck time simply is $t_{p}=\frac{l_{p}}{c}$, so the time it takes for light to travel the Planck length - this is naturally known. All the Planck units seems mystical when calculated from the Newton gravitational constant and the Planck constant. Only when we have to do with output in $\mathrm{kg}$ do we need the Planck constant.

We do not question that the Newton gravitational constant is a universal constant, but it is a composite constant that consists of more fundamental entities. Further, the Planck constant is not even needed if we switch to a more fundamental mass measurement that involves the Planck length and the Planck frequency.

\section{Conclusion}

We have shown that the $\mathrm{kg}$ mass definition is a reduced Compton frequency ratio. It is the reduced Compton frequency of the mass in question divided by the reduced Compton frequency of one kg. We introduce two new mass definitions. One that is simple reduced Compton frequency per Planck second and one that is the reduced Compton frequency per Planck second multiplied by one over the speed of light. Both of these we claim are just as valid as the standard definition of mass. The standard definition of mass does not contain the Planck length and leads to unnecessarily complicated formulas because one has to get rid of the Planck constant to predict most gravity phenomena. From our new mass definition, we have derived from scratch and shown how the Planck length can be found and measured independent of any knowledge of the Planck constant or Newton's gravitational constant. The Newton gravitational constant is a composite constant that is not even unique. It is the Planck length and the reduced Compton frequency of matter, as well as the speed of gravity (equal to the speed of light), that are truly important for gravity. 


\section{References}

[1] A. H. Compton. A quantum theory of the scattering of x-rays by light elements. Physical Review. 21 (5):, 21(5), 1923.

[2] S. Prasannakumar, S. Krishnaveni, and T. K. Umesh. Determination of rest mass energy of the electron by a compton scattering experiment. European Journal of Physics, 33(1), 2012.

[3] D. Dolce and Perali. On the compton clock and the undulatory nature of particle mass in graphene systems. The European Physical Journal Plus, 130(41), 2015.

[4] S. Lan, P. Kuan, B. Estey, D. English, J. M. Brown, M. A. Hohensee, and Müller. A clock directly linking time to a particle's mass. Science, 339, 2013.

[5] Albert Einstein. On the electrodynamics of moving bodies. Annalen der Physik, English translation by George Barker Jeffery 1923, (17), 1905.

[6] E. G. Haug. The gravitational constant and the Planck units. A simplification of the quantum realm. Physics Essays Vol 29, No 4, 2016.

[7] E. G. Haug. The ultimate limits of the relativistic rocket equation. The Planck photon rocket. Acta Astronautica, 136, 2017.

[8] E. G. Haug. Can the Planck length be found independent of big g ? Applied Physics Research, 9(6), 2017.

[9] E. G. Haug. Newton and Einstein's gravity in a new perspective for Planck masses and smaller sized objects. International Journal of Astronomy and Astrophysics, 2018.

[10] E. G. Haug. Modern physics incomplete absurd relativistic mass interpretation. and the simple solution that saves Einstein's formula. Journal of Modern Physics, 9(14), 2018.

[11] M. A. Markov. On gravity and the uncertainty principle. Suppl. Commemoration Issue for 30th Anniversary of the Meson Theory by Dr.H.Yukawa, 14(20), 1965.

[12] E. G. Haug. Revisiting the derivation of Heisenberg's uncertainty principle: The collapse of uncertainty at the Planck scale. preprints.org, 2018.

[13] Isaac Newton. Philosophiae Naturalis Principia Mathematica. London, 1686.

[14] H. Cavendish. Experiments to determine the density of the earth. Philosophical Transactions of the Royal Society of London, (part II), 88, 1798.

[15] E. G. Haug. Planck quantization of newton and Einstein gravitation. International Journal of Astronomy and Astrophysics, 6(2), 2016.

[16] M. E. McCulloch. Gravity from the uncertainty principle. Astrophysics and Space Science, 349(2), 2014.

[17] R.S. Van Dyck, F.L. Moore, D.L. Farnham, and P.B. Schwinberg. New measurement of the proton-electron mass ratio. International Journal of Mass Spectrometry and Ion Processes, 66(3), 1985. 